\title{
Malformações Cardíacas Fetais. Diagnóstico e Conduta
}

\author{
Paulo Zielinsky
}

Porto Alegre, RS

A cardiologia pediátrica contemporânea transformouse radicalmente a partir do advento da ecocardiografia, do cateterismo intervencionista e do avanço das técnicas cirúrgicas. Entretanto, foi a possibilidade de identificar precocemente a presença de malformações cardíacas, ainda durante o desenvolvimento in utero, através da ecocardiografia fetal, que constituiu a pedra angular da trajetória da ciência cardiológica em direção ao futuro. Esta técnica de concepção aparentemente simples, utilizando os princípios já conhecidos pela longa experiência do cardiologista pediátrico com o diagnóstico não invasivo pelo ultra-som, viabilizou o estabelecimento de condutas salvadoras para o concepto cardiopata, antes e logo após o nascimento.

O feto chega ao cardiologista para avaliação porque são identificados durante os exames pré-natais fatores de risco para alterações cardíacas (diabetes materno, uso de drogas teratogênicas, história familiar de cardiopatia congênita, rubéola e outras infecções durante a gestação, presença de anormalidades extracardíacas à ultra-sonografia obstétrica e outros) ${ }^{1}$. Entretanto, é fundamental lembrar que mais de $90 \%$ das malformações cardíacas ocorrem em fetos sem qualquer fator de risco ${ }^{2}$. Portanto, o rastreamento populacional dirigido, durante a ecografia pré-natal de rotina, através da observação sistemática do coração fetal, aliado a um conhecimento básico, por parte do operador, das suas características normais, é o único caminho para que o diagnóstico das cardiopatias congênitas possa ser ampliado, em termos de atenção primária à população.

Nosso objetivo é revisar as principais doenças cardíacas estruturais de apresentação fetal, dentro de um enfoque fisiopatológico, enfatizando o reconhecimento ecocardiográfico, sua repercussão e as opções para o manejo perinatal, não sendo abordadas anormalidades funcionais do coração, como as miocardiopatias ou arritmias, que constituem tópicos de outros artigos.

\section{Cardiopatias fetais com comprometimento funcional tardio}

Este grupo de malformações cardíacas fetais, embora possa estar representado por cardiopatias até complexas,

Unidade de Cardiologia Fetal do Instituto de Cardiologia do Rio Grande do Sul/ Fundação Universitária de Cardiologia - Porto Alegre

Correspondência: Paulo Zielinsky - IC/FUC - Unidade de Pesquisa - Av. Princesa Isabel, 395 - 90620-001 - Porto Alegre, RS não costuma trazer sinais de disfunção durante o período intra-uterino, não mostrando modificações progressivas e não fazendo prever sinais de sofrimento cardiológico no período neonatal imediato. Por isso, são doenças cujo conhecimento pré-natal não altera a conduta obstétrica, no que se refere ao momento e ao local do nascimento, assim como ao tipo de parto previsto. Apesar disso, obviamente é importante seu reconhecimento ecocardiográfico, já que os pacientes necessitarão acompanhamento cardiológico atento durante e após o $1^{\circ}$ mês de vida extra-uterina, de forma a prover terapêutica medicamentosa, intervencionista ou cirúrgica, de acordo com a situação. Não menos importante é a possibilidade da cardiopatia observada, embora sem repercussão funcional significante, representar parte do espectro de uma doença sistêmica fetal mais ampla, como as síndromes genéticas, especialmente as trissomias, ou anomalias extracardíacas graves, tais como a hérnia diafragmática, a onfalocele, as alterações do sistema nervoso central e as uropatias obstrutivas ${ }^{1,3}$. Com o avanço da medicina fetal, muitas destas entidades patológicas são manejadas durante a vida intra-uterina e, assim, o diagnóstico da malformação cardíaca adquire significado especial.

Constituem exemplos deste grupo de cardiopatias aquelas cuja apresentação pós-natal é de hiperfluxo pulmonar, pela presença de curto-circuitos esquerda-direita, como a comunicação interventricular (CIV), o defeito septal atrioventricular, a comunicação interatrial (CIA) e a persistência do canal arterial. As malformações complexas "cianóticas" sem estenose pulmonar, como a dupla via de saída do ventrículo direito (VD), truncus arteriosus, a drenagem venosa pulmonar anômala não obstrutiva e as conexões atrioventriculares univentriculares manifestam-se, precocemente, durante $\mathrm{o} 1^{\circ}$ mês de vida pós-natal, mas não requerem intervenção imediata e, portanto, também pertencem ao grupo em questão. Algumas cardiopatias fetais "obstrutivas", em sua forma menos importante, igualmente podem ser consideradas de apresentação "tardia", como a tetralogia de Fallot e as estenoses aórtica ou pulmonar não críticas.

A comunicação interventricular é facilmente reconhecida à ecocardiografia fetal como uma solução de continuidade na região perimembranosa ou muscular do septo interventricular, de diâmetro variável. Os defeitos pequenos podem passar despercebidos, o que não tem implicações do ponto de vista de manejo perinatal. A diminuição espontânea do diâmetro do orifício e, até o fechamento completo in 
utero, geralmente por aposição de tecido tricúspide acessório às suas bordas, nos defeitos perimembranosos, pode freqüentemente ser documentada por ecocardiogramas (ECO) fetais seriados, com intervalo de poucas semanas (fig. 1) ${ }^{4}$. O mapeamento a cores demonstra ofluxo interventricular, geralmente de baixa velocidade, devido à virtual equiidade de pressões entre os dois ventrículos. Em alguns casos, não é possível a demonstração do curto-circuito, apesar da inequívoca imagem ao estudo bidimensional.

Os defeitos do septo atrioventricular, com todas as suas variantes morfológicas, estão entre as cardiopatias mais facilmente detectadas pelo ECO fetal. Por isso, em todas as séries constituem-se em uma das malformações mais prevalentes ${ }^{5}$. É importante salientar que mais da metade dos casos é acompanhada de outras alterações fetais, tanto

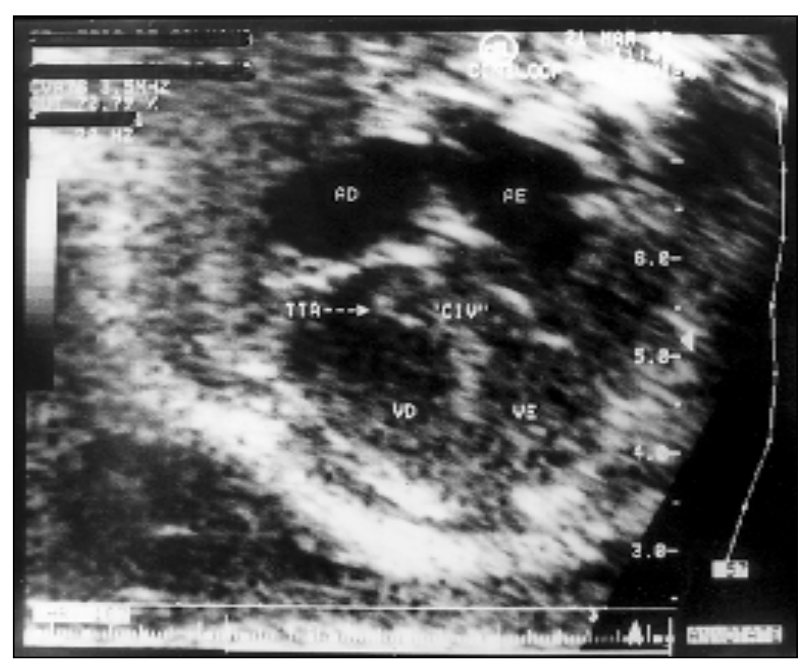

Fig. 1 - Corte de 4-câmaras. Comunicação interventricular perimembranosa, parcialmente fechada por tecido tricúspide acessório. AD-átrio direito; $\mathrm{AE}$-átrio esquerdo; VD- ventrículo direito; VE- ventrículo esquerdo; CIV- comunicação interventricular; TTA- tecido tricúspide acessório.

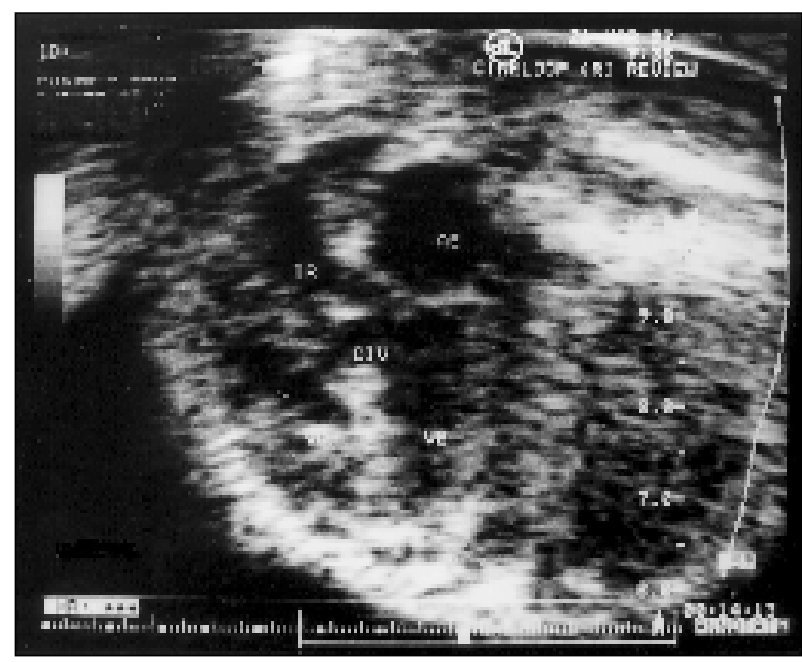

Fig. 2 - Corte de 5-câmaras. Truncus arteriosus. Observa-se a via de saída única do grande vaso truncal, cavalgando o septo trabecular. TR- truncus; AE- átrio esquerdo; CIV- comunicação interventricular; VE- ventrículo esquerdo. cardíacas (dupla via de saída do VD, isomerismo direito ou esquerdo, estenose pulmonar), como extracardíacas, muito especialmente as cromossomopatias. A identificação da valva atrioventricular comum, geralmente com defeito interatrial do tipo ostium primum e freqüentemente com uma CIV de via de entrada, é a marca registrada do defeito. A presença de regurgitação sistólica de um ou ambos os componentes da valva atrioventricular é a regra, sendo sua detecção possível tanto pelo Doppler pulsátil como pelo mapeamento de fluxo a cores.

A comunicação interatrial é difícil de ser diagnosticada com segurança à ecocardiografia fetal de rotina, já que o feto normal possui naturalmente o forame oval, cuja membrana, o septum primum, abaula para o átrio esquerdo (AE) devido ao fluxo interatrial direita-esquerda fisiológico. As situações em que a CIA pode ser identificada ao estudo bidimensional correspondem aos raros casos em que as bordas do orifício são hiper-refringentes e não se visibiliza a membrana do forame oval, ou aos casos de átrio comum, que não apresentam septo atrial ${ }^{1}$. Os defeitos do tipo ostium primum, como já comentados no tópico sobre defeito septal atrioventricular, são facilmente detectados.

A persistência do canal arterial é virtualmente impossível de ser predita pelo ECO fetal, pois a perviabilidade do ducto arterioso é também pré-requisito para uma dinâmica circulatória normal e está presente fisiologicamente. Nos casos de cardiopatias complexas com obstrução completa ao fluxo pulmonar, discutidas no item "Cardiopatias com circulação pulmonar dependente do canal arterial", o canal arterial tem morfologia peculiar, e sua perviabilidade ao nascimento é a regra.

A dupla via de saída do VD é identificada quando o ECO fetal mostrar que a aorta e a artéria pulmonar emergem preferencialmente do VD. Dois tipos morfológicos distintos podem estar presentes, dependendo da posição relativa dos vasos entre si e da relação do vaso posterior com a CIV. Assim, nos casos em que a aorta é anterior, geralmente, a artéria pulmonar está relacionada com a CIV, caracterizando a anomalia de Taussig-Bing quando não houver estenose pulmonar. Seu diagnóstico diferencial com a transposição dos grandes vasos com septo interventricular aberto depende, exclusivamente, do grau de cavalgamento da valva pulmonar sobre o septo trabecular, que é maior que $50 \%$ nos fetos com dupla via de saída do VD. Nas situações em que a CIV é subaórtica, a artéria pulmonar é o vaso anterior, sendo freqüente que o curso das grandes artérias seja lado a lado. Estes fetos não costumam apresentar sofrimento intrauterino e não necessitam, habitualmente, de cuidados cardiológicos imediatos no período perinatal ${ }^{6}$.

O diagnóstico de truncus arteriosus durante a vida fetal não é difícil, já que depende da identificação de um grande vaso cavalgando o septo trabecular, sobre uma ampla CIV (fig. 2). Não se observa artéria pulmonar emergindo 
do coração e, nos casos de truncus tipo I ou II, freqüentemente detecta-se o tronco da artéria pulmonar originandose da aorta ascendente. A valva truncal é muitas vezes anormal e o mapeamento a cores pode mostrar a presença de fluxo diastólico regurgitante através da mesma.

\section{A drenagem venosa pulmonar anômala total não} obstrutiva pode ser demonstrada pelo ECO fetal, mas não se constitui em diagnóstico fácil. Como o volume de fluxo pulmonar é baixo durante a vida intra-uterina, o grau de sobrecarga das câmaras direitas não costuma ser o aspecto mais chamativo. Quando as veias pulmonares são identificadas e sua drenagem é demonstrada em câmara venosa posterior ao $\mathrm{AE}$ (fig. 3) ${ }^{7,8}$, especialmente com o auxílio do mapeamento de fluxo a cores, o grau de certeza aumenta. Da mesma forma, um seio coronário dilatado, em um caso suspeito, dirige o raciocínio para esta possibilidade. Entretanto, as formas mistas de drenagem venosa pulmonar anômala são de difícil avaliação, e necessitarão de minucioso exame pósnatal para a complementação propedêutica.

As conexões atrioventriculares univentriculares, em suas diversas formas de apresentação morfológica, são identificadas com precisão, mesmo durante a avaliação ecográfica rotineira, pela simples observação do corte de 4câmaras. Os casos com ausência de conexão atrioventricular direita (atresia tricúspide) ou esquerda (atresia mitral) mostrarão apenas uma valva atrioventricular, contralateral à conexão ausente. Habitualmente é identificada uma cavidade ventricular rudimentar abaixo da conexão ausente (VD na atresia tricúspide e ventrículo esquerdo (VE) na atresia mitral) ${ }^{8,9}$. A dupla via de entrada para VE, VD ou indeterminado pode estar presente com qualquer modo de conexão atrioventricular (valva atrioventricular comum, duas valvas pérvias, uma valva imperfurada e straddling ou overriding valvar). Como regra, o que se visibiliza com clareza é a presença de um ventrículo grande e um rudimentar, que pode estar à esquerda (mais freqüentemente) ou à direita. $\mathrm{O}$ tipo de conexão ventrículo-arterial é variável, podendo ser concordante, discordante, dupla via de saída ou via de saída única. Quando não há estenose pulmonar, as manifestações pós-natais ocorrem geralmente após a $1^{\circ}$ semana de vida e, assim, são consideradas "tardias" do ponto de vista fetal.

A tetralogia de Fallot é bastante freqüente durante a vida fetal, e seu diagnóstico ecocardiográfico costuma ser realizado sem dificuldades ${ }^{6,8}$. Chama à atenção a presença de uma grande CIV subaórtica, acompanhada usualmente de um aumento do calibre da aorta ascendente, que cavalga o septo trabecular (fig. 4). Este pode ser o primeiro sinal para o observador experimentado. Ao ser buscado o corte transversal, habitualmente pode ser demonstrado que o septo infundibular apresenta desvio ântero-superior, com ou sem obstrução demonstrável à via de saída do VD. De fato, na maior parte das vezes, o fluxo analisado pelo Doppler pulsátil ou pelo mapeamento a cores não demonstra turbulência abaixo ou acima da valva pulmonar, já que a estenose infundibular tende a se desenvolver mais tarde. Obviamente, é a gravidade da estenose pulmonar o árbitro do enquadramento desta entidade patológica no grupo com comprometimento funcional "tardio" ou "neonatal imediato", com conseqüente planejamento da conduta perinatal de acordo com a situação ${ }^{1}$.

A estenose valvar aórtica e a estenose valvar pulmonar, quando não graves, não constituem risco imediato in utero, assim como no período neonatal. Por isso, o diagnóstico ecocardiográfico fetal terá utilidade para orientar o acompanhamento após a alta do berçário, não sendo esperados problemas na primeira semana de vida. A identificação destas lesões é baseada na imagem de uma valva aórtica ou pulmonar espessa, com fusão comissural, exibindo movimento em cúpula na sístole, acompanhada de fluxo transvalvar turbulento, ao Doppler ou ao mapeamento a cores. Nos casos de pequena repercussão, não ocorre aumento da espessura ventricular esquerda ou direita, respectivamente.

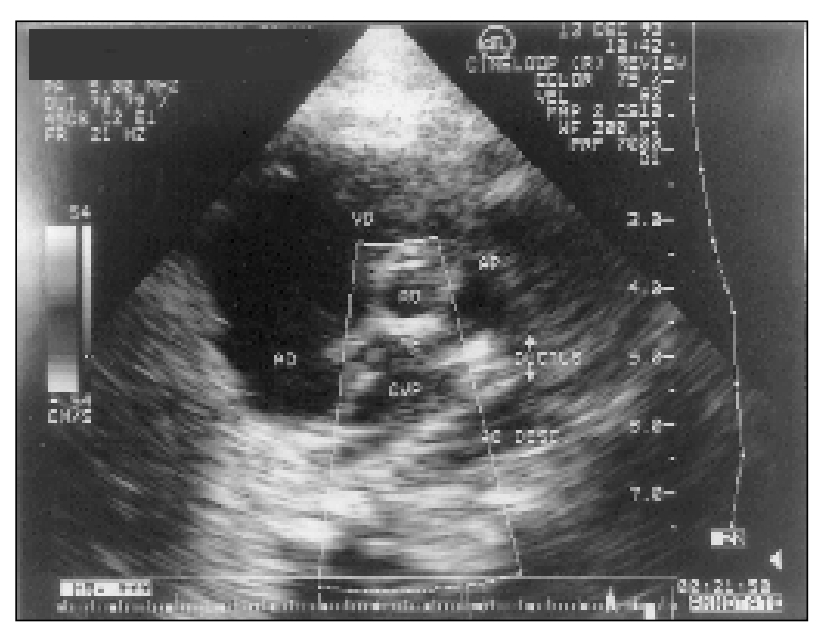

Fig. 3 - Corte transverso basal. Drenagem venosa pulmonar anômala total. Há um lago venoso pulmonar posterior ao átrio esquerdo, que é pequeno. $\mathrm{O}$ ventrículo direito e a artéria pulmonar estão dilatados. CVP- confluência venosa pulmonar; AO- aorta; $\mathrm{AP}$ - artéria pulmonar; $\mathrm{VD}$ - ventrículo direito; $\mathrm{AD}$ átrio direito; $\mathrm{AE}$ - átrio esquerdo; AO desc aorta descendente.

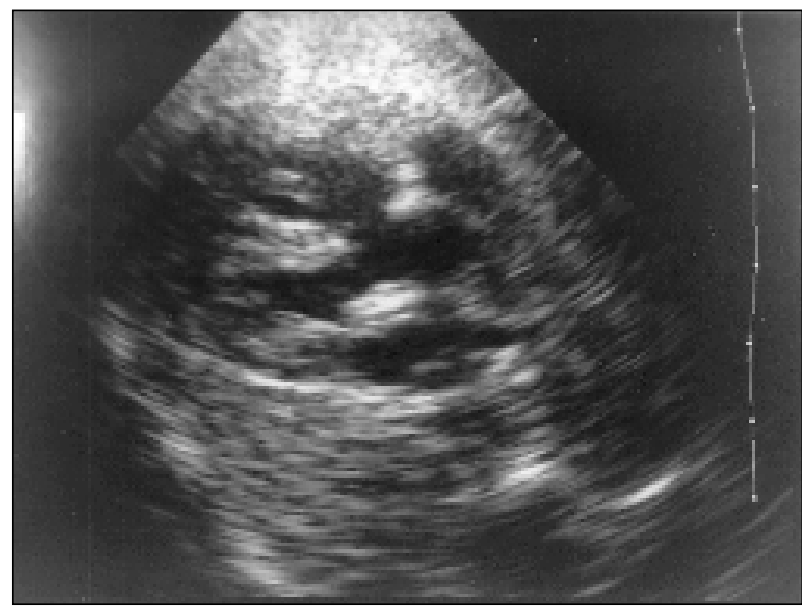

Fig. 4 - Corte longitudinal. Tetralogia de Fallot. Há grande comunicação interventricular e a aorta cavalga o septo trabecular em $50 \%$ do seu anel. 
Entretanto, é importante a lembrança de que, se o diagnóstico ecocardiográfico for realizado no $2^{\circ}$ trimestre de gestação, é fundamental o acompanhamento com exames seriados, para tornar possível a detecção de eventual progressão da gravidade da lesão e conseqüente necessidade de planejar terapêutica neonatal imediata ou mesmo intervenção intra-uterina ${ }^{1}$.

\section{Cardiopatias fetais com comprometimento funcional neonatal}

Este grupo de malformações, apesar de complexas e graves, não costuma apresentar repercussão funcional significante durante a vida intra-uterina, porque o padrão circulatório não se altera a ponto de impedir um adequado funcionamento do sistema cardiovascular fetal. Seu diagnóstico pré-natal, entretanto, é mandatório, para que seja possível equacionar o nascimento em ambiente adequado para o atendimento neonatal, assim como para planejar as ações terapêuticas imediatas. $\mathrm{O}$ transporte intra-uterino do feto com cardiopatia grave, enquanto ele se encontra no melhor ambiente existente, o útero materno, melhora de forma considerável a expectativa de sucesso na terapêutica cardiológica perinatal. O reconhecimento deste grupo de doenças pelo ECO fetal constitui-se na própria essência da cardiologia fetal, e pode representar a linha divisória entre a vida e a morte do concepto cardiopata.

\section{Cardiopatias com circulação pulmonar dependente} do canal arterial - Este grupo de malformações fetais é representado pelas cardiopatias em que existe obstrução crítica ou completa do fluxo do ventrículo venoso para a artéria pulmonar. Assim, todo o sangue que chega aos pulmões, após o nascimento, depende da perviabilidade do canal arterial. Independentemente das variações morfopatológicas presentes, o diagnóstico pré-natal é essencial. A utilização materna de medicamentos que possam causar efeito constritivo sobre o ducto arterioso, como a indometacina, o ácido acetilsalicílico e outros antiinflamatórios não esteróides, pode ser evitada ${ }^{10}$. Além disso, o conhecimento da cardiopatia fetal permite que o parto (cesáreo) seja planejado de forma a permitir a presença da equipe especializada no momento do nascimento, que terá "hora marcada ". A infusão de prostaglandina $\mathrm{E}_{1}$ ou $\mathrm{E}_{2}$, para a manutenção da permeabilidade ductal, assim como as manobras de reanimação cardiorrespiratória e correção da acidose, quando indicadas, podem ser iniciadas imediatamente, antes mesmo do transporte do recém-nascido para a Unidade de Tratamento Intensivo neonatal. A indicação de cirurgias paliativas, como anastomoses sistêmico-pulmonares, ou a abertura da valva pulmonar, no bloco cirúrgico ou na sala de cateterismo, podem então ser equacionadas antes que ocorra deterioração clínica por hipóxia ou acidose, com evidente melhora do prognóstico.

Os exemplos típicos deste subgrupo de malformações são a atresia pulmonar com septo intacto, a estenose valvar pulmonar crítica, a atresia pulmonar com CIV (tetralogia de
Fallot com atresia pulmonar) e as cardiopatias complexas acompanhadas de estenose ou atresia pulmonar (dupla via de saída do VD, conexões atrioventriculares univentriculares, isomerismos atriais).

A atresia pulmonar com septo intacto é uma cardiopatia de fácil identificação à ecocardiografia fetal, já que o sinal mais chamativo é muito evidente: o VDé hipoplásico, com sua cavidade muscularizada, geralmente acompanhado de um átrio direito (AD) aumentado e de uma valva tricúspide claramente anormal. Durante a ecografia obstétrica, também já é possível a constatação de que existe uma grosseira anormalidade ao corte de 4-câmaras e, por isso, a maioria dos casos de atresia pulmonar com septo intacto é encaminhada pelo ultra-sonografista (fig. 5). O ECO identifica, também, o plano da valva pulmonar, que está fechado no corte das vias de saída, e o mapeamento de fluxos a cores demonstra, além da onipresente insuficiência tricúspide, que o fluxo no tronco da artéria pulmonar, junto à valva, é retrógrado, da mesma forma que o fluxo no ducto, que também é reverso, isto é, da esquerda para a direita ${ }^{10}$. Quando a circulação coronária é dependente do VD, podem ser identificados, ao mapeamento a cores, comunicações coronáriocavitárias (sinusóides), embora este seja um achado relativamente pouco freqüente ${ }^{8}$. Nos casos com VD extremamente hipoplásico, com apenas uma porção, este dado deve ser especialmente procurado pelo cardiologista fetal, devido às implicações terapêuticas advindas do seu conhecimento pré-natal. Assim, na presença de um ventrículo pouco desenvolvido, com sinusóides observados ao ECO fetal, o planejamento cirúrgico pós-natal não deverá incluir a abertura da valva pulmonar e, portanto, o neonato poderá ser encaminhado para anastomose sistêmico-pulmonar sem a necessidade de cateterismo cardíaco, a não ser que a CIA seja restritiva e que uma atriosseptostomia esteja indicada.

A estenose valvar pulmonar crítica também costuma mostrar ao ECO fetal um VD cuja cavidade está diminuída,

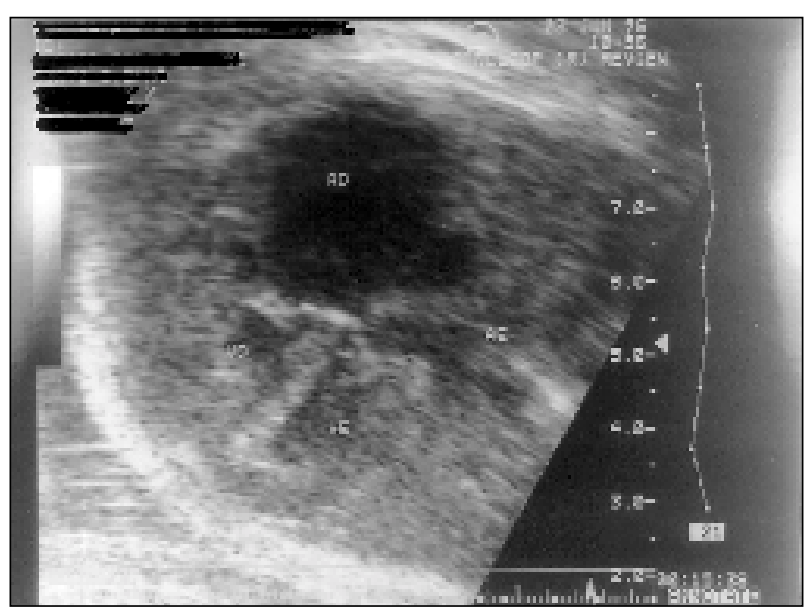

Fig. 5 - Corte de 4-câmaras. Atresia pulmonar com septo intacto. $\mathrm{O}$ átrio direito está grandemente aumentado e o septo interatrial abaula inteiramente para a esquerda. $\mathrm{AD}$ - átrio direito; VD- ventrículo direito; $\mathrm{AE}$ - átrio esquerdo; VE- ventrículo esquerdo. 
com acentuada hipertrofia de suas paredes, associado a um aumento do AD e a uma valva tricúspide insuficiente. Entretanto, pode-se identificar uma valva pulmonar que, embora importantemente estenótica, mostra mobilidade sistólica e fluxo transvalvar turbulento, geralmente representado por jato de alta velocidade, demonstrado ao Doppler e ao mapeamento a cores. No que se refere ao planejamento terapêutico, a conduta neonatal imediata, além das medidas gerais já comentadas, incluirá a dilatação percutânea com balão da valva pulmonar. Nos casos em que o orifício seja diminuto, sua ampliação com radiofrequiência poderá ser considerada durante o cateterismo. Por isso, novamente se enfatiza o benefício do diagnóstico pré-natal.

Os fetos portadores de atresia pulmonar com $\mathbf{C I V}$ mostram ao estudo ecocardiográfico muitos dos achados característicos da tetralogia de Fallot, como aumento de calibre da aorta ascendente, cavalgamento da valva aórtica sobre o septo trabecular, CIV subaórtica com mau-alinhamento septal e desvio ântero-superior do septo infundibular. Entretanto, a condição básica para o seu diagnóstico é a demonstração de uma via de saída do VD fechada, sem fluxo anterógrado e sem uma valva pulmonar detectável. No tronco da artéria pulmonar, quando presente, o fluxo costuma ser retrógrado. Em muitos casos, a presença de circulação colateral representada por vasos sistêmico-pulmonares, especialmente com origem na aorta, pode ser identificada ${ }^{11}$. O canal arterial costuma apresentar calibre diminuído em relação ao normal e, freqüentemente, apresenta um trajeto tortuoso ${ }^{10}$. Como em todos os casos com circulação pulmonar ducto-dependente, também se observa fluxo sistólico esquerda-direita e ausência de fluxo diastólico no canal, refletindo a alta resistência pulmonar durante a vida fetal. Estes fetos deverão também receber atenção imediata, de forma a planejar a melhor abordagem neonatal, com cateterismo precoce e cirurgia paliativa a curto prazo.

As cardiopatias complexas com atresia pulmonar comportam-se, do ponto de vista ecocardiográfico, de modo semelhante ao já abordado, mas com o agravante de apresentarem obstrução crítica ou completa ao fluxo pulmonar e, assim, dependerem da perviablidade do canal arterial para a perfusão dos pulmões ${ }^{10}$. $\mathrm{O}$ manejo perinatal estará baseado na adequada definição morfológica e funcional da doença e, obviamente, da variante anatômica detectada durante a vida fetal. Na maioria das vezes, o neonato receberá prostaglandina logo após o nascimento e será submetido a uma anastomose sistêmico-pulmonar nos primeiros dias de vida.

Cardiopatias com circulaçãosistêmica dependente do canal arterial - As malformações cardíacas fetais deste subgrupo correspondem às obstruções graves ao fluxo arterial sistêmico: síndrome da hipoplasia do coração esquerdo, coarctação da aorta e interrupção do arco aórtico. Apresentam como característica comum a dependência da circulação sistêmica à perviabilidade do ducto arterioso. Por isso, durante a vida fetal, não costumam apresentar comprometimento funcional significante. Entretanto, logo após o nascimento, quando ocorre a constrição fisiológica do canal arterial, os neonatos manifestam importante sofrimento, com importante congestão pulmonar e falência circulatória, evoluindo rapidamente para o óbito se não forem tomadas medidas imediatas. Por esta razão, o diagnóstico pré-natal constitui-se em uma necessidade imperiosa para a modificação do prognóstico, ao permitir o transporte intra-uterino e o manejo perinatal com prostaglandina previamente à indicação cirúrgica. $\mathrm{O}$ impacto do diagnóstico de obstruções críticas do VE durante a vida fetal já foi amplamente demonstrado ${ }^{12}$.

A síndrome da hipoplasia do coração esquerdo é uma das cardiopatias fetais de mais fácil diagnóstico, já que a grosseira diminuição da cavidade ventricular esquerda é evidente ao corte de 4-câmaras, mesmo para o observador menos experiente (fig. 6). Durante o rastreamento ultrasonográfico obstétrico, a desproporção de tamanho dos ventrículos é geralmente detectada, com conseqüente encaminhamento da gestante para ecocardiografia fetal. Alguns fatores de risco para hipoplasia do coração esquerdo são a presença de síndrome de Turner e a história familiar de cardiopatia congênita obstrutiva, como estenose aórtica, coarctação, interrupção do arco aórtico e a própria hipoplasia do coração esquerdo. Além da cavidade ventricular esquerda hipoplásica, observa-se hiper-refringência endocárdica relacionada à presença de fibroelastose ${ }^{8}$. Existe atresia aórtica e mitral, com uma aorta ascendente de calibre diminuto e ausência de fluxo anterógrado através das valvas aórtica e mitral. Freqüentemente, o forame oval é pequeno e restritivo. O fluxo da aorta ascendente é reverso, já que depende exclusivamente do canal arterial, que costuma ser grande. Também nesta cardiopatia é importante o acompanhamento ecocardiográfico fetal seriado, já que muitas vezes um exame realizado com menos de 20 semanas pode

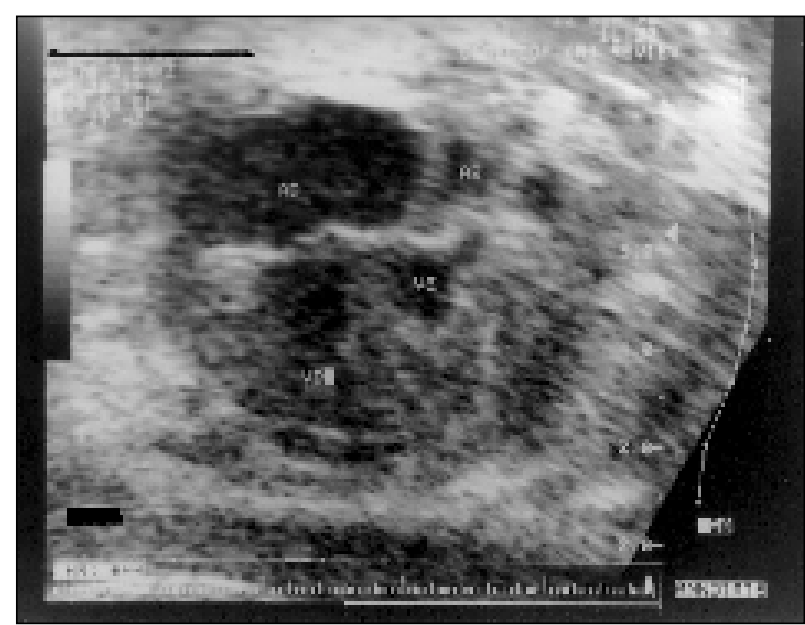

Fig. 6 - Corte de 4-câmaras. Síndrome da hipoplasia do coração esquerdo. Observase a desproporção de tamanho entre os dois ventrículos, com a cavidade ventricular esquerda hipoplásica. $\mathrm{AD}$ - átrio direito; $\mathrm{AE}$ - átrio esquerdo; VE- ventrículo esquerdo; VD- ventrículo direito. 
mostrar apenas um VE de forma mais arredondada, endocárdio brilhante e com contração anormal e que, ao longo das próximas semanas de gestação, vai gradativamente mostrando o aparecimento dos sinais característicos da síndrome do coração esquerdo hipoplásico ${ }^{9}$. O conhecimento pré-natal da hipoplasia do coração esquerdo reveste-se de especial importância, pela possibilidade de buscar doadores potenciais para eventual transplante cardíaco neonatal, que é hoje o tratamento cirúrgico de escolha para esta situação. A cirurgia de Norwood, embora se constitua na única alternativa cirúrgica ao transplante neonatal, apresenta ainda resultados desalentadores a longo prazo, na maioria dos centros. Uma vez obtido um doador compatível, se a maturidade pulmonar fetal o permitir, o nascimento pode ser acelerado e o transplante realizado imediatamente.

A coarctação da aorta, se importante, é uma das cardiopatias de apresentação neonatal capaz de desenvolver os mais graves quadros de insuficiência cardíaca (IC) e colapso circulatório, sendo a circulação sistêmica dependente da perviabilidade do canal arterial ${ }^{13}$. Durante a vida

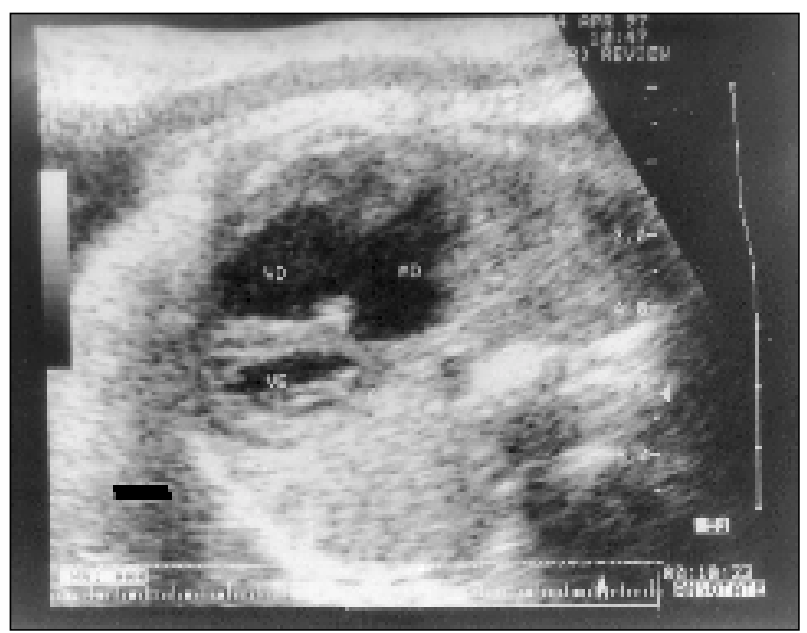

Fig. 7 - Corte de 4-câmaras. Coarctação da aorta. O ventrículo direito apresenta importante aumento em relação ao esquerdo. VD- ventrículo direito; AD-átrio direito; VEventrículo esquerdo.

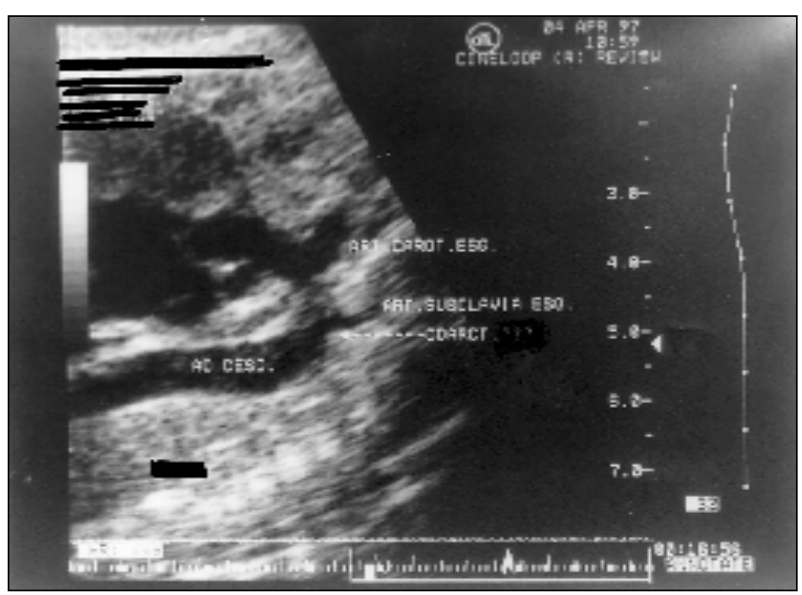

Fig. 8 - Corte do arco aórtico. Coarctação da aorta. A região ístmica é estreita, mas a aorta descendente é calibrosa. fetal, entretanto, não existe o substrato para a falência cardiocirculatória, já que o canal amplamente aberto permite que o VD leve à aorta descendente o débito sistêmico. Do ponto de vista diagnóstico, a coarctação aórtica é de difícil reconhecimento ecocardiográfico intra-uterino, devendo a suspeita ser baseada em alguns dados propedêuticos indiretos ${ }^{14}$. Assim, o aspecto mais chamativo é a desproporção de tamanho entre os dois ventrículos, sendo o VD maior e mais hipertrófico que o esquerdo (fig. 7). Da mesma forma, a artéria pulmonar costuma estar dilatada, sendo de calibre muito maior do que a aorta ascendente. Por outro lado, a aorta descendente é calibrosa, a partir da inserção do canal arterial (fig. 8). Não raramente, existe uma hipoplasia relativa da aorta ascendente e, às vezes, é possível identificar um estreitamento localizado após a emergência da artéria subclávia esquerda, embora esse achado não possa ser a condição para o diagnóstico (fig. 9). O mapeamento de fluxo a cores mostra em muitos casos a presença de um fluxo reverso (do istmo para os vasos braquiocefálicos e a valva aórtica) na aorta ascendente ou do $\mathrm{AE}$ para o $\mathrm{AD}$, quando o estreitamento é importante. Quando existe CIV, freqüentemente esta é perimembranosa, com algum cavalgamento da aorta sobre o septo trabecular. Deve ser reiterado que o diagnóstico de coarctação aórtica constitui-se ainda no "calcanhar de Aquiles" da ecocardiografia fetal, especialmente nos casos menos graves.

A interrupção do arco aórtico não é um diagnóstico freqüente durante a vida fetal. Devido à ampla perviabilidade do canal arterial, os sinais de repercussão funcional só são manifestos após a constrição pós-natal do ductus. $\mathrm{O}$ estudo ecocardiográfico fetal mostra uma imagem do arco ductal que é em tudo semelhante à observada no feto normal, com a aorta descendente continuando o trajeto do canal arterial a partir da artéria pulmonar. $\mathrm{O}$ que pode chamar à atenção do examinador é a presença de uma artéria subclávia esquerda, ou de uma carótida e uma subclávia, originando-se da porção distal do arco ductal, logo após a inserção do calibroso canal na aorta descendente. Além disso, está quase sempre presente uma grande CIV de via de saída, na maioria das vezes com mau-alinhamento septal por desvio posterior do septo infundibular, o que é bastante característico da associação com a interrupção do arco aórtico.

\section{Cardiopatias com circulações pulmonar e sistêmica} em paralelo - O exemplo característico deste subgrupo de malformações fetais é a transposição dos grandes vasos, em que existe discordância ventrículo-arterial com conexão atrioventricular concordante. Como a aorta se origina do VD e a artéria pulmonar do VE, as circulações pulmonar e sistêmica, no período pós-natal, estão em paralelo, e a saturação sistêmica depende exclusivamente do grau de mistura entre as duas circulações, isto é, da magnitude da comunicação interatrial, da perviabilidade do canal arterial ou da eventual presença de uma CIV. Assim, no período neonatal o recém-nascido apresenta precocemente sofrimento hipó- 


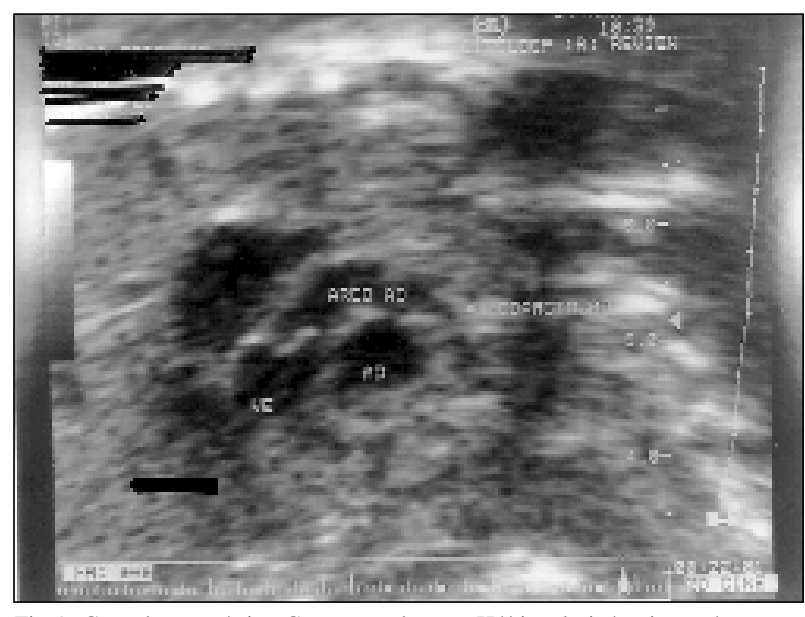

Fig. 9- Corte do arco aórtico. Coarctação da aorta. Há hipoplasia ístmica, após a emergência da artéria subclávia esquerda. AD- átrio direito; VE- ventrículo esquerdo.

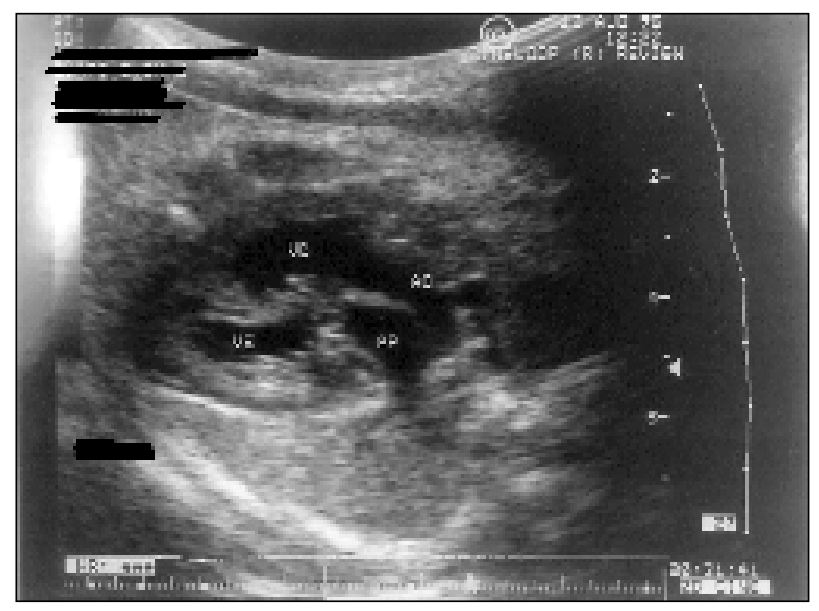

Fig. 10 - Corte longitudinal. Transposição dos grandes vasos. A aorta origina-se à frente, do ventrículo direito, e a artéria pulmonar, posterior, do ventrículo esquerdo. VD- ventrículo direito; AO- aorta; VE- ventrículo esquerdo; AP- artéria pulmonar.

xico, dependendo de medidas clínicas e cirúrgicas urgentes para evitar a acidose, melhorar a hipoxemia e corrigir o defeito ${ }^{13}$. Entretanto, durante a vida fetal, não ocorre qualquer comprometimento funcional, já que a circulação pulmonaré escassa, de alta resistência e pouco dependente do tipo de conexão ventrículo-arterial. A importância do diagnóstico pré-natal da transposição dos grandes vasos está na necessidade de atendimento neonatal imediato, com a utilização de prostaglandina para manter volume ventricular esquerdo aumentado e melhorar a saturação, avaliar oportunidade e indicação eventual de atriosseptostomia com balão e, principalmente, preparar o neonato para a cirurgia de Jatene, hoje universalmente o procedimento de escolha para a correção deste defeito. O reconhecimento ecocardiográfico intra-uterino da transposição dos grandes vasos está baseado na identificação da artéria pulmonar como vaso posterior com sua característica bifurcação, originando-se do VE, e da aorta como vaso anterior, com origem no VD (fig. 10) ${ }^{6}$. A presença de defeitos associados, como a CIV, é também avaliada. Quando a artéria pulmonar tem calibre reduzido, menor que o da aorta, pode-se suspeitar da concomitância de estenose pulmonar.
Cardiopatias com obstrução ao retorno venoso pulmonar - A drenagem venosa pulmonar anômala total infradiafragmática obstrutiva, entidade relativamente rara e de diagnóstico difícil durante a vida intra-uterina, deve estar na mente do cardiologista fetal quando diante de um feto com aumento do VD em relação ao VE, em que se identifica uma confluência venosa pulmonar junto ao AE sem comunicação com o mesmo, especialmente ao se utilizar o mapeamento a cores com baixa frequiência de repetição de pulso (PRF), e uma veia vertical com fluxo descendente, em direção ao fígado ${ }^{7}$. Às vezes, é possível demonstrar a presença de um lago venoso anormal próximo às veias hepáticas, freqüentemente com um fluxo contínuo de alta velocidade na sua comunicação com o sistema porta. Esta cardiopatia, embora bem tolerada no período fetal, rapidamente ocasiona deterioração clínica grave no período neonatal imediato, com congestão pulmonar importante e hipoxemia, necessitando de tratamento cirúrgico urgente. Por esta razão, o nascimento do bebê no próprio local onde será prestado o atendimento neonatal é mandatório.

\section{Cardiopatias fetais com comprometimento funcional intra-uterino}

Este grupo de malformações cardíacas fetais é aquele que tem a maior perspectiva de ser beneficiado com o avanço das técnicas invasivas de terapêutica pré-natal, já que suas manifestações clínicas ocorrem durante a vida intrauterina. Embora inúmeras cardiopatias com grave repercussão in utero ainda não possam ser manipuladas diretamente por métodos intervencionistas, o conhecimento dessas doenças permite o tratamento medicamentoso, por via transplacentária (materna) ou por cordocentese (fetal direta), conforme a indicação específica ${ }^{15,16}$. Obviamente, a detecção de malformações cardíacas fetais com potencial para comprometimento funcional intra-uterino deve ser imediatamente seguida do encaminhamento da gestante para internação em ambiente hospitalar apropriado para tratamento fetal, com capacidade para atendimento por equipe multidisciplinar, preferentemente composta por cardiologista fetal, obstetra, neonatologista, eletrofisiologista, cirurgião cardíaco, enfermeiro, psicólogo e assistente social ${ }^{1}$. Desta forma, o equacionamento da conduta poderá ser discutido com a família, com o objetivo de definir qual a opção mais apropriada para aquele feto em particular, considerando aspectos técnicos, morais e éticos.

A anomalia de Ebstein da valva tricúspide é uma das malformações de pior prognóstico durante a vida fetal. Quando se apresenta na sua forma grave, com importante displasia e deslocamento caudal da valva tricúspide, geralmente existe maciça regurgitação em direção à porção atrializada do VD e ao $\mathrm{AD}^{17}$. Existe grande cardiomegalia, porque o tamanho do AE costuma ser extremamente aumentado, sendo este o achado que chama a atenção do ultrasonografista e que o faz encaminhar a gestante para ecocardiografia fetal (fig. 11). É frequiente a presença de sinais de 
IC fetal, com hidropisia, caracterizada por ascite, derrame pleural e pericárdico, edema de pele e de couro cabeludo ${ }^{18}$. O índice cardiotorácico está grandemente aumentado, o que por si só já faz antever a presença de hipoplasia pulmonar significativa, com consequiente e previsível sofrimento hipóxico neonatal. Muitas vezes existe estenose ou atresia pulmonar associadas e, não raramente, o estudo ecocardiográfico seriado durante a vida fetal permite a observação do desenvolvimento progressivo de atresia pulmonar "funcional", em que a ausência de fluxo anterógrado pela valva pulmonar, decorrente da grave regurgitação tricúspide, faz com que todo o débito sistólico do VD tenha um curso retrógrado para o AD. A evolução para o óbito intra-uterino é freqüente e este fato deve ser claramente exposto aos pais. Entretanto, quando é possível atingir um estágio da gestação em que seja possível acelerar a maturidade pulmonar fetal, com a utilização de corticosteróides do tipo betametasona ou dexametasona, a cesárea deve ser praticada, para a tentativa de terapêutica cirúrgica pós-natal. $\mathrm{O}$ uso de surfactantes pode melhorar as chances do neonato, mas quando existe hipoplasia pulmonar o curso é geralmente fatal. As possibilidades cirúrgicas no período neonatal são o transplante cardíaco ou o fechamento do anel tricúspide e da artéria pulmonar associados a uma derivação cavopulmonar parcial. Com ambas as técnicas, os resultados no momento não são alentadores. No que se refere ao tratamento medicamentoso durante a vida fetal, são utilizados o digital e os diuréticos, por via materna, no sentido de diminuir os efeitos da congestão sistêmica. Quando a anomalia de Ebstein se acompanha de taquiarritmias, como o flutter atrial e a taquicardia supraventricular, são utilizados o digital e outros antiarrítmicos, como a amiodarona e o sotalol por via transplacentária (fig. 12) ${ }^{19}$. A eventual necessidade de cordocentese para introdução de antiarrítmicos ao feto diretamente deve ser balanceada com o risco geral do concepto, já que este costuma ter sua reserva funcional muito diminuída.

A estenose aórtica crítica durante a vida fetal é uma doença extremamente grave, com alto risco de morte intrauterina por IC e baixo débito, especialmente nos casos em que o VE apresenta hipocontratilidade acentuada e fibroelastose endocárdica secundária. O ECO fetal mostra que o anel valvar é reduzido e a valva aórtica apresenta importante espessamento e diminuição da sua mobilidade (fig. 13) ${ }^{8}$. O mapeamento a cores demonstra a turbulência do fluxo sistólico, mas com freqüência não é registrado um gradiente alto ao Doppler, devido ao importante déficit funcional contrátil do VE. Esta cavidade apresenta-se dilatada, com hiper-refringência endocárdica e, na maioria dos casos, é possível a detecção de insuficiência mitral associada, ao Doppler e ao mapeamento a cores, devido à alteração na geometria ventricular esquerda e à dilatação do anel. Os sinais de hidropisia fetal, decorrentes da instalação precoce de IC, são evidentes e, na maioria das vezes, acentuados, com ascite, derrame pleural e pericárdico, edema de couro cabeludo, de pele e de tecidos moles ${ }^{20}$. A conduta terapêu- tica depende da idade gestacional e da maturidade pulmonar fetal. Quando o feto for maduro, obviamente está indicada a interrupção da gestação, em hospital preparado para tratamento intervencionista ou cirúrgico imediato ${ }^{12}$. Entretanto, infelizmente muitos casos apresentam deterioração precoce durante a vida fetal, ainda durante o $2^{\circ}$ trimestre, em que não se pode alimentar a expectativa de ser obtida maturação pulmonar suficiente para permitir sobrevida neonatal, mesmo após administração de corticosteróides à gestante no período antecedente à possível interrupção. Nessa situação, a par do tratamento medicamentoso tradicional (especialmente diuréticos em doses altas), pode ser considerada a possibilidade de intervenção intra-uterina, com a finalidade de tentar a dilatação da valva aórtica com cateter balão introduzido através de uma agulha posicionada junto ao anel aórtico, após punção do ápex do VE, sob visão ecocardiográfica ${ }^{15,16,21,22}$. Este procedimento, de alta complexidade, ainda deve ser encarado como experimental, embora já tenha sido realizado em seis fetos (quatro no Guy's Hospital de Londres, um no Hospital das Clínicas

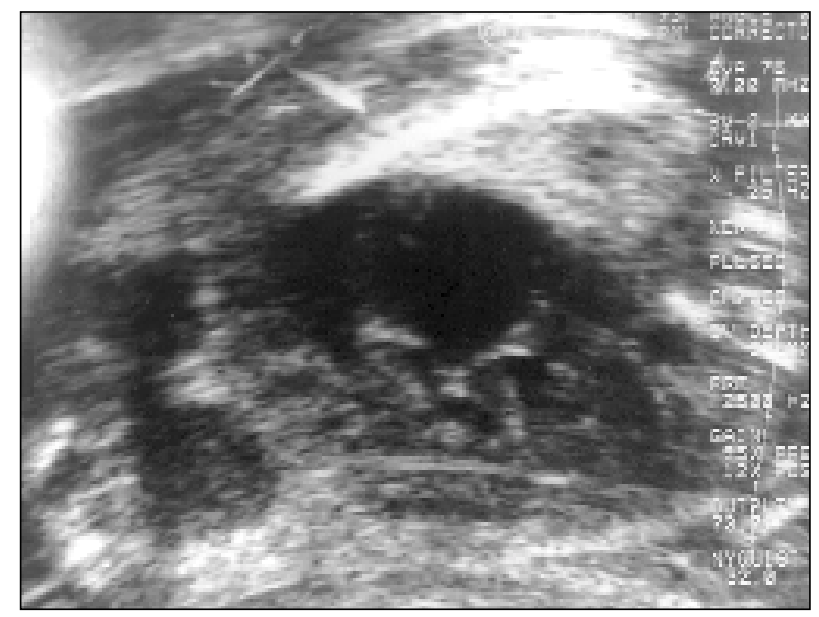

Fig. 11 - Corte de 4-câmaras. Anomalia de Ebstein. Observa-se que a valva tricúspide é displásica, redundante e implantada caudalmente. $\mathrm{O}$ átrio direito é muito aumentado.

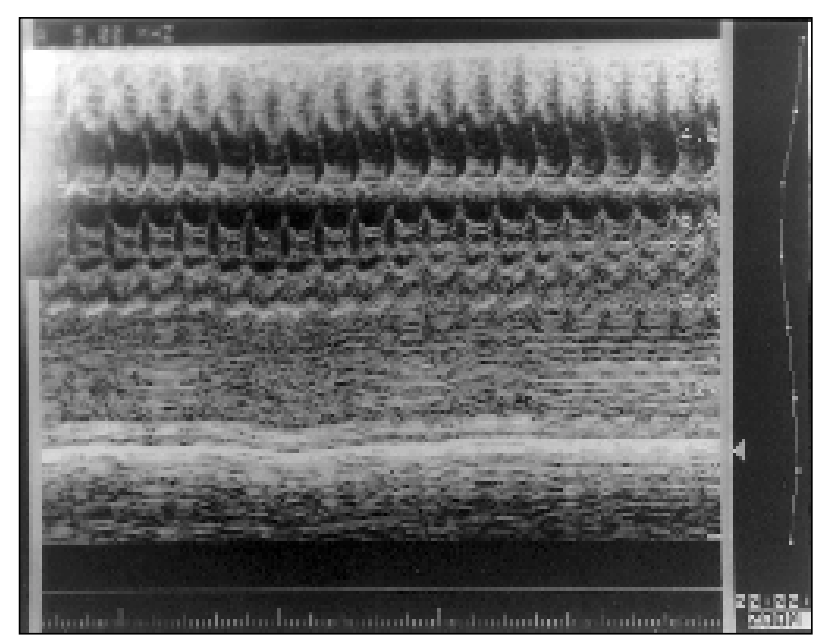

Fig. 12 - Ecocardiograma modo-M. Taquicardia supraventricular sustentada em feto com anomalia de Ebstein. A condução atrioventricularé 1:1 e a frequiência cardíaca é de $240 \mathrm{bpm}$ 
de São Paulo e um no Instituto de Cardiologia do Rio Grande do Sul). Nessas séries, em apenas um caso houve relato de sobrevida tardia, mas se este resultado é cotejado com o prognóstico sem intervenção, em que a mortalidade fetal ou neonatalé de $100 \%$, justifica-se a idéia do prosseguimento da pesquisa na direção do tratamento intervencionista intra-uterino ${ }^{23}$.

As malformações complexas com isomerismo esquerdo e bloqueio atrioventricular (BAV) total podem apresentar comprometimento funcional grave in utero, devido à freqüência cardíaca baixa. $\mathrm{O}$ prognóstico de $\mathrm{BAV}$ completo associado a cardiopatias complexas é sombrio, com uma mortalidade de $85 \%{ }^{19,24,25}$. Por isso, a importância do diagnóstico pré-natal correto é óbvia. A detecção deste distúrbio da condução atrioventricular baseia-se na observação, ao ECO unidimensional, de atividade ventricular dissociada da atrial, com bradicardia freqüentemente significante. É usual o aparecimento de hidropisia fetal que, quando presente, faz antever um desfecho fatal, caso não sejam tomadas medidas urgentes. Também nesta situação, a interrupção da gestação para implante de marcapasso no período neonatal imediato depende da maturidade pulmonar fetal. Nos casos em que a IC é precoce e grave, com bradicardia acentuada e imaturidade pulmonar documentada por estudo do líquido amniótico, a possibilidade de estimulação elétrica artificial do coração fetal pode ser avaliada ${ }^{26}$. Ainda não há respostas concretas para a questão do implante intra-uterino de marcapasso. Já foi descrita tentativa de introdução de cateter eletródio por via percutânea e um feto hidrópico com BAV, que faleceu após 4 h de estimulação. Nosso grupo implantou um marcapasso epicárdico "a céu aberto", com histerotomia e exposição do tórax fetal, mas não foi possível obter resposta contrátil adequada ao estímulo, pois o feto foi intervido já em estado agônico ${ }^{27}$. Há diversos estudos experimentais em andamento, alguns no Brasil, que buscam a solução para este angustiante problema, mas o momento ainda é de mais dúvidas do que certezas ${ }^{28,29}$.

Oforame oval restritivo, embora não se constitua em uma anomalia estrutural verdadeira, mas a uma diminuição do orifício de passagem do fluxo interatrial, pode causar sofrimento fetal importante, com IC grave ${ }^{30}$. O sinal mais chamativo ao ecocardiografista é a grande dilatação das câmaras direitas, geralmente com insuficiência tricúspide significativa, muitas vezes acompanhado de alteração na dinâmica contrátil do VD. O septum primum abaula para o AE de forma "aneurismática”, sem a mobilidade cíclica característica. A análise do forame oval mostra, ao mapeamento a cores, turbulência do fluxo e aumento da velocidade ao Doppler. O tratamento clínico com dose alta de diurético é geralmente suficiente para a resolução da hidropisia, mas no feto maduro, com piora progressiva, a gestação deve ser interrompida, pois no período pós-natal a situação hemodinâmica favorece o desaparecimento dos sinais de IC.

A constrição do ducto arterioso também não é uma

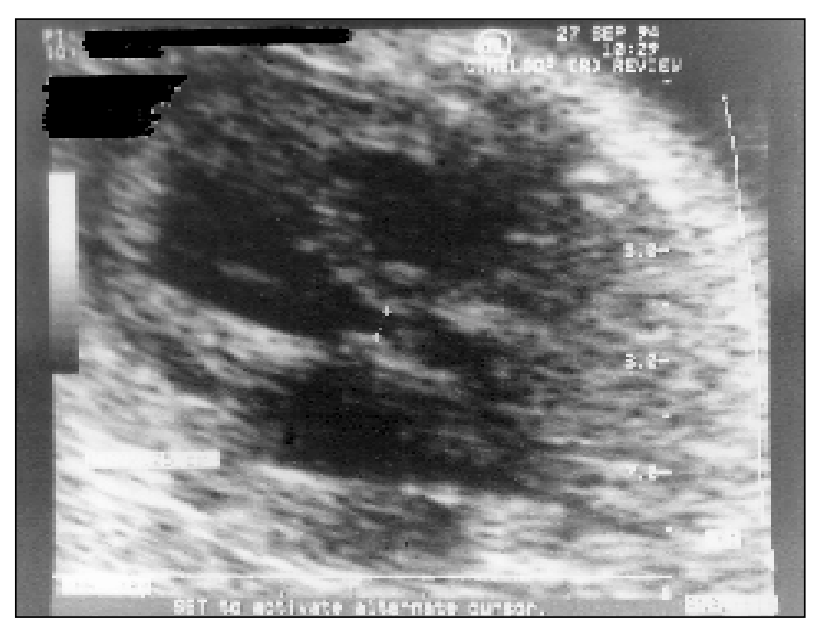

Fig. 13 - Corte longitudinal. Estenose aórtica crítica. O anel valvar é reduzido e a cavidade ventricular esquerda dilatada e hipocontrátil, com fibroelastose endocárdica.

malformação estrutural, mas a possibilidade de causar comprometimento funcional durante a vida fetal deve fazer com que seja uma entidade lembrada diante de um quadro de IC fetal. Como praticamente todo o débito ventricular direito para a circulação sistêmica faz-se através do ducto, um estreitamento do mesmo pode causar importante sobrecarga pressórica do VD e conseqüente hidropisia fetal. A causa mais comum desta entidade é a utilização materna de medicamentos inibidores da prostaglandina E, como os antiinflamatórios não-esteróides, dos quais a indometacina é o exemplo mais tradicional ${ }^{31}$. O ECO fetal identifica com clareza não só o calibre diminuído do canal, como documenta, ao mapeamento a cores, o fluxo turbulento através do mesmo. A análise do fluxo ao Doppler permite mostrar um aumento das velocidades sistólica e diastólica no ducto, servindo este método como parâmetro para avaliar a evolução do feto após a suspensão da indometacina, tenha ela sido administrada para tratar trabalho de parto prematuro ou polihidrâmnio. É muito comum a presença de insuficiência tricúspide, representando a sobrecarga pressórica do $\mathrm{VD}^{32}$. O tratamento desta situação, além da suspensão de drogas potencialmente envolvidas em sua gênese, como a indometacina, passa pela administração de diuréticos, para o combate às manifestações de hidropisia. Nos casos graves, sem resposta à medicação, com maturidade pulmonar fetal, está indicada a interrupção da gestação para tratamento pósnatal, devendo ser cuidadosamente monitorizada a possibilidade de desenvolvimento de hipertensão pulmonar no período neonatal imediato.

\section{Conclusão}

O diagnóstico da maioria das malformações cardíacas fetais é possível de ser realizado ao ECO fetal, sem maiores dificuldades. Entretanto, como já foi enfatizado, é fundamental que o conhecimento básico do coração normal e patológico seja estendido aos ultra-sonografistas obstétricos. Durante a ecografia rotineira, a observação de um achado potencialmente anormal deve levar o examinador a enca- 
minhar a gestante para centro especializado, onde o ECO fetal com mapeamento a cores confirmará ou afastará a suspeita de cardiopatia. Se o diagnóstico de uma malformação cardíaca for estabelecido, a conduta terapêutica dependerá do local de atendimento, do comprometimento funcional atual, potencial ou previsível e da maturidade fetal. A par do tratamento clínico medicamentoso, o transporte intrauterino do feto, o planejamento do atendimento perinatal ou a intervenção intra-uterina poderão ser equacionados ${ }^{33}$.

Os avanços diagnósticos e terapêuticos da cardiologia fetal contemporânea estão caminhando lado a lado com o desenvolvimento de inúmeras correntes de pensamento, que buscam respostas a questões técnicas, científicas, éticas, morais, legais, religiosas e emocionais. Em última análise, o médico que se relaciona com um paciente protegido peloútero materno precisa questionar-se incessantemente se suas atitudes e habilidades estão ou não trazendo perspectivas de maior bem-estar ou de felicidade ao feto e sua família. Se a resposta naquele instante for positiva, a busca das fronteiras da vida pode transpor os limites do impossível.

\section{Referências}

1. Zielinsky $\mathrm{P}$ - Abordagem diagnóstica e terapêutica pré-natal das anormalidades cardíacas fetais. Rev Bras Ecocardio 1992; 17: 10-24.

2. Copel JA, Pilug G, Green J, Hobbins JC, Kleinmann CS - Fetal echocardiographic screening for congenital heart disease: the importance of the four-chanber view. Am J Obstet Gynecol 1987; 157: 648-55.

3. Allan LD, Sharland GK, Lockhkart S, Maxwell DJ - Chromossomal anomalies in fetal congenital heart disease. Ultrasound Obstet Gynecol 1991; 1: 8-11.

4. Zielinsky P, Hagemann LL - Aspectos morfológicos e evolutivos da comunicação interventricular no feto humano: um estudo ecocardiográfico prénatal. Arq Bras Cardiol 1990; 55: B-181.

5. Allan LD, Sharland GK, Milburn A et al - Prospective diagnosis of 1006 consecutive cases of congenital heart disease in the fetus. J Am Coll Cardiol 1994; 23 1452-8.

6. Paladini D, Rustico M, Todros T et al - Conotruncal anomalies in prenatal life. Ultrasound Obstet Gynecol 1996; 8: 241-51.

7. Yeager SB, Parness IA, Spevak PJ, Hornberger LK, Sanders SP - Prenatal echocardiographic diagnosis of pulmonary and systemic venous anomalies. Am Heart J 1994; 128: 397-405.

8. Allan L, Sharland GK, Cook A - Color Atlas of Fetal Cardiology. London: Mosby-Wolfe Publ 1994: 157.

9. Allan LD, Anderson RH, Cook AC - Atresia or absence of the left-side atrioventricuar connection in the fetus: echocardiographic diagnosis and outcome. Ultrasound Obstet Gynecol 1996; 8: 295-302.

10. Mielke G, Steil E, Kendizorratt, Goelz R - Ductus arteriosus-dependent pulmonary circulation secondary to cardiac malformations in fetal life. Ultrasound Obstet Gynecol 1997; 9: 25-9.

11. Zielinsky P, Rossi $\mathrm{F}^{\circ} \mathrm{RI}$, Horowitz ES, Rossi MB - Atresia pulmonar com septo interventricular aberto no feto: diagnóstico ecocardiográfico pré-natal e correlação anátomo-patológica. Rev Bras Ecocardio 1990; 3: 28-9.

12. Chang AC, Huhta JC, Yoon GY et al - Diagnosis, transport and outcome in fetuses with left ventricular outflow tract obstruction. J Thorac Cardiovasc Surg 1991; 102: 841-8.

13. Zielinsky P - Cardiopatias Congênitas. In: Miura E, Pricianoy R Neonatalogia.Princípios e Práticas. $2^{a}$ ed. Porto Alegre: Artes Médicas, 1997: 176.

14. Sharland GK, Chan K, Allan LD - Coarctation of the aorta: difficulties in prenatal diagnosis. Br Heart J 1994; 71: 70-5.

15. Dillenburg RF, Zimmer LP, Frantz N et al - Procedimentos diagnósticos e terapêuticos invasivos durante a vida fetal. Rev AMRIGS 1995; 39: 291-6.

16. Dillenburg RF, Zimmer LP, Frantz N et al - Medicina fetal invasiva: estado da arte. Femina 1996; 24: 203-7.

17. Lang D, Oberkoffer R, Cook A et al - Pathologic spectrum of malformations of the tricuspid valve in prenatal and neonatal life. J Am Coll Cardiol 1991; 17: 11617.

18. Hornberger LK, Sahn DJ, Kleinman CS, Copel JA, Reed KL - Tricuspid valve disease with significant tricuspid insufficiency in the fetus: diagnosis and outcome. J Am Coll Cardiol 1991; 17: 167-73.

19. Zielinsky P - Distúrbios do ritmo cardíaco fetal: detecção e conduta pré-natal. Arq Bras Cardiol 1996; 66: 83-6.

20. Sharland GK, Chita SK, Fagg NIK et al - Left ventricular disfunction in the fetus: relation to aortic valve anomalies and endocardial fibroelastosis. Br Heart J 1991; 66: 419-24.

21. Maxwell D, Allan LD, Tynan M - Balloon dilatation of the aortic valve in the fetus: a report of two cases. Br Heart J 1991; 65: 256-8.

22. Lopes LM, Cha SC, Kajita LJ, Aiello VD, Jatene A, Zugaib M-Balloon dilatation of the aortic valve in the fetus: a case report. Fetal Diagn Ther 1996; 11: 296-300.

23. Allan LD, Maxwell DJ, Carminatti M, Tynan MJ - Survival after fetal aortic balloon valvoplasty. Ultrasound Obstet Gyencol 1995; 5: 90-1.

24. Machado MVL, Tynan MJ, Curry PVL, Allan LD-Fetal complete heart block. Br Heart J 1988; 60: 512-5.

25. Schmidt KG, Ulmer HE, Silverman NH, Kleinmann CS, Copel JA-Perinatal outcome of fetal complete atrioventricular block: a multicenter experience. J Am Coll Cardiol 1991; 91: 1360-6.

26. Carpenter RJ, Strasburger JF, Garson A, Smith RT, Russel L-Fetal ventricular pacing for hydrops secondary to complete atrioventricular block. J Am Coll Cardiol 1986; 8: 1434-6.

27. Zimmer LP, Silva APD, Andrade A, Dillenburg RF, Mendonça E, Zielinsky P Manejo intra-uterino e perinatal do bloqueio atrioventricular total no feto. Arq Bras Cardiol 1996; 67: 11-5.

28. Scagliotti EI, Shimokocil DD, Pringle KG - Permanent cardiac pacemaker implant in the fetal lamb. Pace 1987; 10: 1253-6.

29. Assad RS, Jatene MB, Moreira LPP et al - Fetal heart block: a new experimental model to assess fetal pacing. Pace 1994; 17:1256-63.

30. Zielinksy P, Dillenburg RF, Zimmer LP - Forame oval restritivo: uma causa de insuficiência cardíaca fetal. Rev Bras Ecocardio 1996; 25:12-15.

31. Hukta JC, Cohen AW, Wood DC-Premature constriction of the ductus arteriosus. J Am Soc Echo 1990; 3: 30-4.

32. Mielke G, Penkert U, Krapp M, Schneider-Pungs J, Gembruch U - Fetal and transient neonatal right heart dilatation with severe tricuspid valve insufficiency in association with abnormally S-Shaped Kinking of the ductus arteriosus. Ultrasound Obstet Gynecol 1995; 5: 338-41.

33. Zielinsky P - Cardiopatias congênitas: abordagem pré-natal. In: Gomes MF, Azeredo MAV, Frison LI, Vitola D et al. Rotinas em Cardiologia. Porto Alegre: Artes Médicas, 1996: 274. 